Case Report

\title{
Scleralfixatedintra-ocularlens : A case report
}

\section{Vijay Pai ${ }^{1}$, Jayaram Shetty ${ }^{2}$, Hrishikesh $\operatorname{Amin}^{3} \&$ Samatha P. Shetty ${ }^{4}$}

${ }^{1,3}$ Professors, ${ }^{2}$ Professor \& HOD, ${ }^{4}$ Junior Resident, Department of Ophthalmology, K.S. Hegde Medical Academy, Nitte University, Mangalore, Karnataka, India.

Correspondence

Vijay Pai

Professor, Department of Ophthalmology, K. S. Hegde M edical Academy, Nitte University, Mangalore - 575 018, Karnataka, India. Mobile : +919845278406 E-mail : eyevijay@yahoo.com

\section{Abstract}

In the surgical management of aphakia in cases with deficient posterior capsule ${ }^{1}$, we have described this particular technique which is cost effective and technically less challenging at the same time with satisfactory results.

Keywords: Scleral fixated IOL, SFIOL, Intraocular lens, Aphakia, Secondary IOL

\section{Introduction}

In recent times, Scleral Fixated Intra Ocular Lens (SFIOLs) are preferred over Anterior Chamber IOLs in the surgical management of aphakia in cases with deficient or absent posterior capsule. Historically, various techniques have been described for SFIOL using sutures, glues or techniques where just the tucking of the haptics of the IOL is done without the use of sutures or glue. ${ }^{1,8,9,10,11,12}$

In this particular case we would like to describe a novel technique to perform SFIOL without the need for any specialized instrumentation.

\section{Case History}

A 72 yr old gentlemanpresented in the eye OPD with hypermature cataract with secondary glaucoma. Prior to this he had gradual, painless, progressive decrease in vision in the same eye since more than 6 years. He was a known case of Diabetes M ellitus, Hypertension and Ischemic Heart Disease.

His Best Corrected Visual acuity in the right eye was Perception of Light with Access this article online Quick Response Code

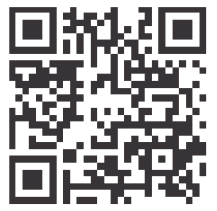
accurate Projection of Rays and in left eye was 6/18. Slit I a m p examination of his RE showed ciliary congestion, microcystic corneal oedema, shallow anterior chamber, mild flare, pseudoexfoliative material at the pupillary margin, sluggishly reacting pupil and hypermature cataract. On further examination, phacodonesis along with iridodonesis was noted. Left eye examination on slit lamp was unremarkable except for immature senile cataract. Intraocular pressurewas $60 \mathrm{mmHg}$ in the right eye and $16 \mathrm{mmHg}$ in the left eye by Goldmannapplanation tonometry.

Based on the clinical findings a diagnosis of Right eye hypermature subluxated cataract with pseudoexfoliation and secondary glaucoma and Left eyesenile immature cataract wasmade.

After medical control of intraocular pressure, subsequent day lens extraction with anterior vitrectomy was performed under $L A$. To facilitate the delivery of lens, radial iridotomy was done at 12 'o' clock.

At the time of discharge his best correctedvisual acuity was 6/60 and IOP was under control. Patient was re admitted 5 weeks later wherein Right eye scleral fixated IOL with anterior vitrectomy and pupilloplastywas done under LA (Fig.1)

Two scleral pockets (Fig.2) approximately $3 \mathrm{~mm} * 3 \mathrm{~mm}$ in dimension were created at 3 and 9 'o' clock limbus. Scleral tunnel from previous surgery was re-opened. Two clear corneal side port incisions were made a 2 and 10 'o' clock. Anterior vitrectomy was performed. 10-0 prolene suture with one end straight needle and the other end micro point 
spatula, was passed at 3 ' 0 ' clock and brought out at 9'o' clock by rail road technique ${ }^{3}$ (Fig.3) using a $1 \frac{1 / 2}{2}$ inch 26 guage needle. The suture was pulled using a sinskey hook through the main wound and was cut. The two cut ends were sutured to the eyelets in the haptics of the SFIOL. The IOL was positioned behind the iris, knots were secured in the scleral bed and the scleral flaps sutured using 10-0 nylon. The radial iridotomy was sutured using 10-0 prolene by Siepser knot, ${ }^{4}$. (Fig.4) Anterior vitrectomy was performed again using Triamcinolone as a guide and the main wound was sutured with single 10-0 nylon suture.

Post operatively, the vision was $6 / 60$ and the patient had mild anterior chamber reaction with IOP of $28 \mathrm{mmHg}$ which was managed medically. At the end of first week, the Best Corrected Visual Acuity improved to 6/12 and IOL was well centred(Fig.5) with an intraocular pressure of $16 \mathrm{mmHg}$ by Goldmannapplanation tonometry.

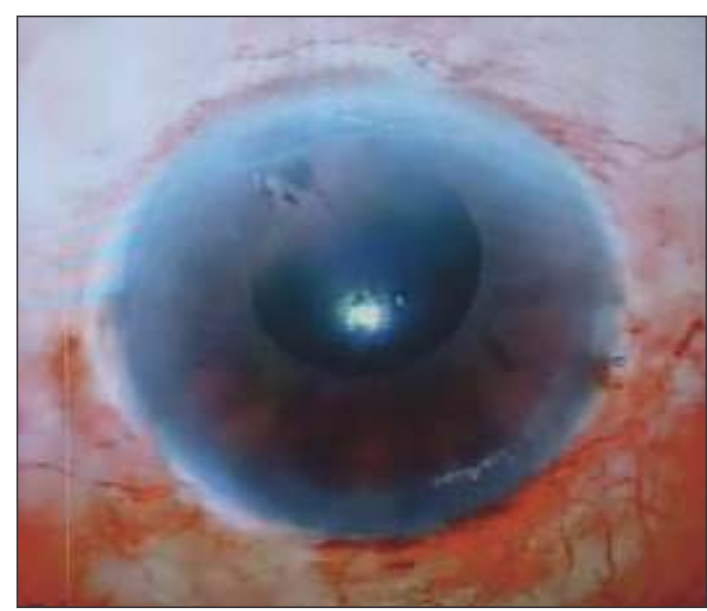

Fig. 1 : Pre-operative status

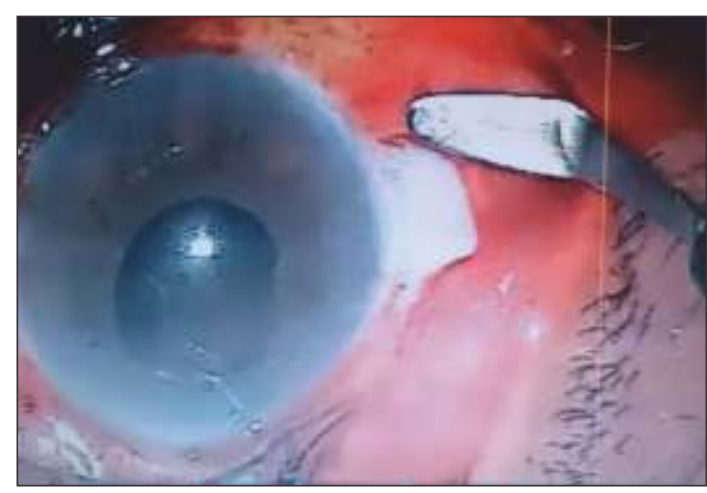

Fig. 2 : Creating a scleral flap at 9'o'clock

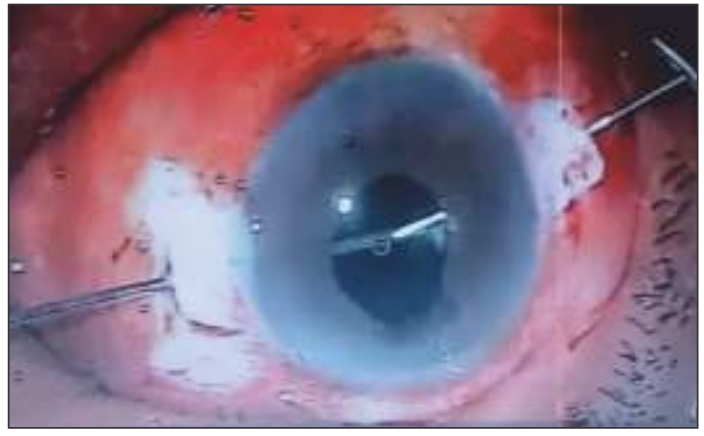

Fig.3 : Rail road technique

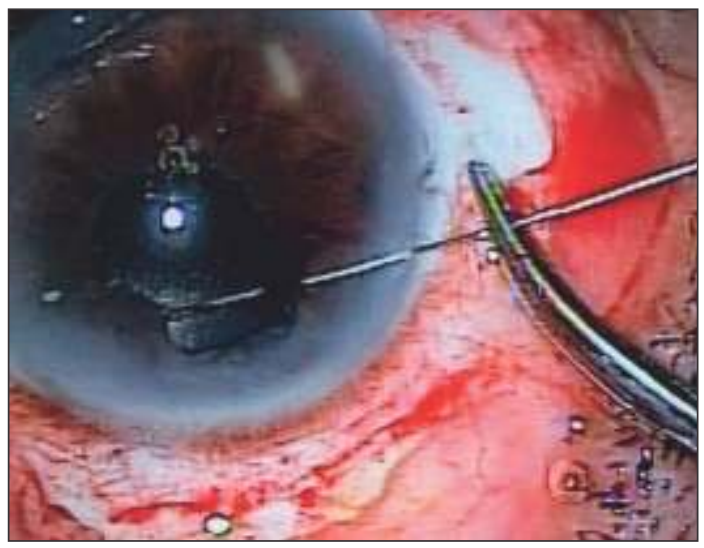

Fig. 4 : Siepser knot

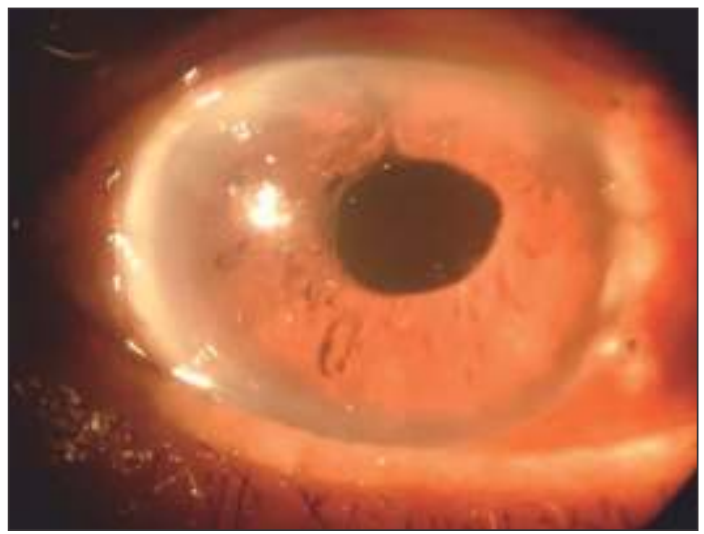

Fig. 5 : Final outcome with SFIOL in place

\section{Discussion}

Since the introduction of IOLs in the late 1940s by Sir Harold Ridley, there have been a lot of changes in the designs, fixation and indications for its use ${ }^{6}$. Intact posterior capsule is mandatory for routine posterior chamber IOL (PCIOL) implantation ${ }^{7}$. Anterior chamber IOLs have been tried in the absence of posterior capsule but, have been noted to carry high risk of post operative complications. Some surgeons suggested suturing a posterior chamber lens to the iris, but complications such as iris chafing, uveitis, and 
pupillary constriction were noted. To avoid these complications, methods to transsclerally fixate posterior chamber lenses to the ciliary sulcus have been developed ${ }^{6}$.

SFIOLs were first reported by Malbran ${ }^{8}$ and co-authors in patients who had previous intracapsular cataract extraction. Later on, various alternatives using different kinds of sutures, needles and IOL designed have been suggested by surgeonsworld-over.

Technique for suturelessintrascleral fixation of PCIOLs was first reported by M aggi and M aggi, ${ }^{90}$. Gabor and Pavlidis ${ }^{11}$ described a technique for sulcus fixation of the haptics of the IOL. Use of a biological glue to attach the haptic to sclera was a technique described by Agarwal. ${ }^{12,13}$

It is recommended to measure the white to white distance to avoid IOL decentration due to disparity between the globe and IOL. Flexible materials such as polypropylene and polymethylmethacrylate with low resistance to deformation are recommended for the haptics of the IOL. This reduces the risk of haptic fracture during surgery.

\section{References}

1. Por Y.M., Lavin M.J. Techniques of Intraocular Lens Suspension in the Absence of Capsular/Zonular Support. Survey of Ophthalmology , Volume 50, Issue 5, 429-462

2. Lee V Y, Yuen H K, Kwok A K. Comparison of outcomes of primary and secondary implantation of scleral fixated posterior chamber intraocular lens. BrJ Ophthalmol 2003;87:1459-1462

3. D K Berler and M A Friedberg. Scleral fixation of posterior chamber intraocular lens implants combined with vitrectomy

TransAm Ophthalmol Soc. 1991; 89: 215-234

4. S. Siepser, MD, Closed-Chamber Slipping Suture Technique for Iris Repair. EyeWorld, July 1997, page 3

5. David F. Chang, Siepser slipknot for M cCannel iris-suture fixation of subluxated intraocular lenses. J Cataract Refract Surg 2004; 30:1170-1176

6. Shapiro A, Leen MM. External transscleral posterior chamber lens fixation. Arch Ophthalmol. 1991; 109: 1759-60.

7. Apple DJ, Brems RN, Park RB, Kavk-Van Norman D, et al Anterior chamber lenses. Part I Complications and pathology and a review of designs. Cataract 'Refract Surg 1987,13:157-174
Scleral fixated IOLs have the advantages of remote positioning from corneal endothelium and proximity to the nodal point and rotational axis of the eye.

It is necessary to appropriately position the IOLs to prevent complications such as pigment dispersion, raised IOP, intraocular haemorrhage and cystoid macular edema. Scleral fixation of IOLs are also associated with decreased corneal endothelial cell density, IOL tilt, decentration and dislocation, vitreous haemorrhage, post-operative refractive errors, infection and retinal detachment ${ }^{9}$. Another disadvantage is the longer surgical time required foritsfixation.

\section{Conclusion}

Scleral fixated PCIOL implantation provide favourable visual outcomes in cases with inadequate capsular or zonular support. Needle guided intrascleral fixation of the IOL is possible using instruments used in standard cataract surgery. However, it requires a longer duration of follow up.

8. M albran, ES, M albran, EJr, and Negri, I. Lens guide suture for transport and fixation of secondary intraocular lens implantation after intracapsular extraction. IntOphthalmol. 1986; 9: 151-160

9. Rodri'guez-Agirretxe I, Acera-Osa A, Ubeda-Erviti M. Needle-guided intrascleral fixation of posterior chamber intraocular lens for aphakia correction. J Cataract Refract Surg 2009; 35:2051-2053

10. Maggi, R. and Maggi, C. Sutureless scleral fixation of intraocular lenses. J Cataract Refract Surg. 1997; 23: 1289-1294

11.Gabor, S.G. and Pavlidis, M.M. Suturelessintrascleral posterior chamber intraocular lens fixation. I Cataract Refract Surg. 2007; 33: 1851-1854

12. Kumar DA, Agarwal A, Prakash D, Jacob S, Agarwal A. Glued Intrascleral Fixation of Posterior Chamber Intraocular Lens in Children. Am J Ophthalmol, Volume 153, Issue 4, 594-601.e2

13. Kumar DA, Agarwal A, Jacob S, Agarwal A. Glued Trans-scleral Intraocular Lens Exchange for Anterior Chamber Lenses in Complicated Eyes: Analysis of Indications and Results. Am J Ophthalmol, Volume 156, Issue 6, 1125 - 1133.e2 